MATHEMATICS OF COMPUTATION

Volume 70 , Number 234 , Pages $863-872$

S $0025-5718(00) 01215-1$

Article electronically published on February 17, 2000

\title{
FINDING STRONG PSEUDOPRIMES TO SEVERAL BASES
}

\author{
ZHENXIANG ZHANG \\ Dedicated to the memory of P. Erdös (1913-1996)
}

\begin{abstract}
Define $\psi_{m}$ to be the smallest strong pseudoprime to all the first $m$ prime bases. If we know the exact value of $\psi_{m}$, we will have, for integers $n<\psi_{m}$, a deterministic primality testing algorithm which is not only easier to implement but also faster than either the Jacobi sum test or the elliptic curve test. Thanks to Pomerance et al. and Jaeschke, $\psi_{m}$ are known for $1 \leq m \leq 8$. Upper bounds for $\psi_{9}, \psi_{10}$ and $\psi_{11}$ were given by Jaeschke.

In this paper we tabulate all strong pseudoprimes (spsp's) $n<10^{24}$ to the first ten prime bases $2,3, \cdots, 29$, which have the form $n=p q$ with $p, q$ odd primes and $q-1=k(p-1), k=2,3,4$. There are in total 44 such numbers, six of which are also $\operatorname{spsp}(31)$, and three numbers are spsp's to both bases 31 and 37. As a result the upper bounds for $\psi_{10}$ and $\psi_{11}$ are lowered from 28- and 29-decimal-digit numbers to 22-decimal-digit numbers, and a 24-decimal-digit upper bound for $\psi_{12}$ is obtained. The main tools used in our methods are the biquadratic residue characters and cubic residue characters. We propose necessary conditions for $n$ to be a strong pseudoprime to one or to several prime bases. Comparisons of effectiveness with both Jaeschke's and Arnault's methods are given.
\end{abstract}

\section{INTRODUCTION}

If $n$ is prime, then (as Fermat knew) the congruence

$$
b^{n-1} \equiv 1 \bmod n
$$

holds for every $b$ with $\operatorname{gcd}(n, b)=1$. In general, if (1.1) holds, then we say that $n$ passes the Fermat (pseudoprime) test to base $b$; if, in addition, $n$ is composite, then we call $n$ a pseudoprime to base $b$ (or $\operatorname{psp}(b)$ for short). It is well-known that for each base $b$, there are infinitely many $\operatorname{psp}(b)$ 's. There are odd composites $n$, called Carmichael numbers, which are pseudoprimes to every base relatively prime to $n$. Alford, Granville and Pomerance [2] proved that there are infinitely many Carmichael numbers.

For these reasons, in some cases it will be difficult to find proofs of compositeness using the Fermat test (1.1). A stronger form of the test does much better. Write

Received by the editor August 1, 1997 and, in revised form, June 22, 1998 and May 24, 1999. 2000 Mathematics Subject Classification. Primary 11Y11, 11A15, 11A51.

Key words and phrases. Strong pseudoprimes, Rabin-Miller test, biquadratic residue characters, cubic residue characters, Chinese remainder theorem.

Supported by the China State Educational Commission Science Foundation and by NSF of China Grant 10071001. 
$n-1=2^{s} d$ with $d$ odd. If $n$ is prime, then

(1.2) either $b^{d} \equiv 1(\bmod n)$ or $b^{2^{r} d} \equiv-1(\bmod n)$ for some $r=0,1, \cdots, s-1$

holds for every $b$ with $\operatorname{gcd}(n, b)=1$. If $(1.2)$ holds then we say that $n$ passes the Miller (strong pseudoprime) test [12] to base $b$; if, in addition, $n$ is composite, then we say $n$ is a strong pseudoprime to base $b, \operatorname{or} \operatorname{spsp}(b)$ for short. Monier [13] and Rabin [15] proved that if $n$ is an odd compoite positive integer, then $n$ passes the Miller test for at most $(n-1) / 4$ bases $b$ with $1 \leq b \leq n-1$. Thus the RabinMiller test appeared: given a positive integer $n$, pick $k$ different positive integers less than $n$ and perform Miller test on $n$ for each of these bases; if $n$ is composite the probability that $n$ passes all $k$ tests is less than $1 / 4^{k}$.

Define $\psi_{m}$ to be the smallest strong pseudoprime to all the first $m$ prime bases. If $n<\psi_{m}$, then only $m$ Miller tests are needed to find out whether $n$ is prime or not. This means that if we know the exact value of $\psi_{m}$, then for integers $n<\psi_{m}$ we will have a deterministic primality testing algorithm which is not only easier to implement but also faster than either the Jacobi sum test [1, 6, 7, 8, or the elliptic curve test [5]. From Alford et al. [3] we know that, for any $m$, the function $\psi_{m}$ exists.

From Pomerance et al. [14] and Jaeschke [11] we know the exact value of $\psi_{m}$ for $1 \leq m \leq 8$ and the following facts:

$$
\begin{aligned}
\psi_{9} \leq M_{9} & =41234316135705689041=4540612081 \cdot 9081224161, \\
\psi_{10} \leq M_{10} & =1553360566073143205541002401(28 \text { digits }) \\
& =22754930352733 \cdot 68264791058197, \\
\psi_{11} \leq M_{11} & =56897193526942024370326972321(29 \text { digits }) \\
& =137716125329053 \cdot 413148375987157 .
\end{aligned}
$$

Jaeschke [11 tabulated all strong pseudoprimes $<10^{12}$ to the bases 2,3 , and 5 . There are in total 101 of them. Among these 101 numbers there are 75 numbers $n$ having the form

$$
n=p q \text { with } p, q \text { odd primes and } q-1=k(p-1), k=2,3,4 .
$$

For short we call strong pseudoprimes having the form (1.3) K2-, K3- or K4-spsp's according as $k=2,3$, or 4 .

In this paper we tabulate all strong pseudoprimes $n<10^{24}$ to the first ten prime bases $2,3, \cdots, 29$ which have the form (1.3). There are in total 44 such numbers, among which six numbers are also spsp(31)'s, and three numbers are spsp's to both bases 31 and 37 . As a result the upper bounds for $\psi_{10}$ and $\psi_{11}$ are considerably lowered:

$$
\begin{aligned}
\psi_{10} \leq N_{10} & =1955097530374556503981 \text { (22 digits) } \\
& =31265776261 \cdot 62531552521, \\
\psi_{11} \leq N_{11} & =7395010240794120709381 \text { (22 digits) } \\
& =60807114061 \cdot 121614228121,
\end{aligned}
$$

and a 24-digit upper bound for $\psi_{12}$ is obtained:

$$
\begin{aligned}
\psi_{12} \leq N_{12} & =318665857834031151167461 \text { (24 digits) } \\
& =399165290211 \cdot 798330580441 .
\end{aligned}
$$

The three integers $N_{10}, N_{11}$, and $N_{12}$, should have the same style as integers $M_{9}, M_{10}$, and $M_{11}$, (above), i.e., insert a small space for each 5 digits. The main 
tools used in finding these numbers are biquadratic residue characters and cubic residue characters defined in certain Euclidean domains which are larger than the integer ring $\mathbb{Z}$. Let $D$ be such a domain and $\alpha, \beta, \pi \in D$. If $\pi$ is a nonunit and such that if $\pi \mid \alpha \beta$ then either $\pi \mid \alpha$ or $\pi \mid \beta$, then $\pi$ is called irreducible. By a prime we will always mean a positive prime of $\mathbb{Z}$. Note that a number $n$ having the form (1.3) is determined by a prime $q$, and the prime $q$ is determined by an irreducible $\pi$. We propose necessary conditions on $\pi$ for $n$ to be a strong pseudoprime to one prime base or to the first several prime bases. Thus we have a certain number of candidates $n$ (determined by candidates for irreducibles) strong pseudoprimes at hand. Then we subject these candidates $n$ to Miller's tests, and obtain the desired numbers.

Arnault 4 used a sufficient condition for finding K2-spsp's and successfully found a 337-digit K2-spsp to all the prime bases $<200$. But his condition is too stringent for most K2-spsp's to satisfy. Examples found by the condition are usually much larger than the corresponding $\psi_{m}$. Our bounds $N_{10}, N_{11}$ and $N_{12}$ could not be found by Arnault's condition. Jaeschke 11] used Jacobi sysbols (quadratic residue characters) as his main tools for finding large K2- and K3-spsp's; thus his methods are less effective than ours. See Remarks 3.1 and 3.2 for comparisons in details.

Notation. Let $r$ be a prime and $b$ a positive integer with $r \nmid b$. Denote by $\operatorname{ord}_{r}(b)$ the order of $b$ in the group $\mathbb{Z}_{r}^{*}$. We write $v_{2}(x)=s$ iff $2^{s} \mid x$ and $2^{s+1} \nmid x$ for $x$ a positive integer.

With the above notation we state a lemma which is fundamental for our methods.

Lemma 1.1 (a part of [11, Proposition 1]). Let $n, p, q, k$ be as in (1.3), and let $b$ be a positive integer. If $n$ is an spsp $(b)$, then $v_{2}\left(\operatorname{ord}_{p}(b)\right)=v_{2}\left(\operatorname{ord}_{q}(b)\right)$.

In $\S 2$ we recall and state some basic facts concerning biquadratic residue characters, which are necessary in $\S \S 3$ and 4 , where we describe methods for finding K2- and K4-spsp's. Note that the three bounds $N_{10}, N_{11}$ and $N_{12}$ are all K2-spsp's which are found in $\S 3$. In $\S 5$ we recall and state some basic facts concerning cubic residue characters and describe a method for finding K3-spsp's. All K2-, K3- and K4-spsp's $<10^{24}$ to the first 10 or 9 prime bases are tabulated.

\section{Biquadratic Residue characters}

Throughout this section and the following two sections $D$ denotes the ring $\mathbb{Z}[i]$ of Gaussian integers. It is well-known that $D$ is a Euclidean domain. Let $\alpha, \beta, \pi \in D$. The norm of $\alpha, N(\alpha)=\alpha \bar{\alpha}=1$ iff $\alpha$ is a unit. The units of $D$ are $\pm 1, \pm i$. The irreducibles of $D$ are $\pm 1 \pm i$ with norm 2 , primes $\equiv 3 \bmod 4$ and their associates, and non-real elements with prime norms $\equiv 1 \bmod 4$. A prime $\equiv 1 \bmod 4 \operatorname{must}$ be the norm of an irreducible of $D$. A nonunit $\alpha$ is called primary if $\alpha \equiv 1$ or $3+2 i$ mod 4. Among four associates of a nonunit $\alpha$ satisfying $(1+i) \nmid \alpha$ there is (only) one which is primary.

If $\pi$ is an irreducible with $N(\pi) \neq 2$, then there exists a unique integer $m, 0 \leq$ $m \leq 3$, such that $\alpha^{(N(\pi)-1) / 4} \equiv i^{m} \bmod \pi$. The biquadratic residue character of $\alpha$, for $\pi \nmid \alpha$, is defined and denoted by $\left(\frac{\alpha}{\pi}\right)_{4}=i^{m}$, which is $1,-1, i$ or $-i$. If $\pi \mid \alpha$, then $\left(\frac{\alpha}{\pi}\right)_{4}=0$. If $b$ is an odd prime $\equiv 3 \bmod 4$, then

$$
\left(\frac{\alpha}{\pi}\right)_{4} \equiv \alpha^{\left(b^{2}-1\right) / 4} \bmod b .
$$


Let $\pi=a+b i$ and $\beta=c+d i$ be relatively prime primary irreducibles. Then (the general law of biquadratic reciprocity [10] Theorem 9.2])

$$
\left(\frac{\beta}{\pi}\right)_{4}=\left(\frac{\pi}{\beta}\right)_{4}(-1)^{\frac{N(\beta)-1}{4} \frac{N(\pi)-1}{4}}=\left(\frac{\pi}{\beta}\right)_{4}(-1)^{\frac{a-1}{2} \frac{c-1}{2}} .
$$

Lemma 2.1. Let $\pi$ be primary irreducible with prime $q=N(\pi) \equiv 1 \bmod 4$. Let $b \in \mathbb{Z}$ with $q \nmid b$. Then we have

(I) [10, Lemma 9.10.1]. $\left(\frac{b}{\pi}\right)_{4}=1$ iff $x^{4} \equiv b \bmod q$ has a solution with $x \in \mathbb{Z}$ (b is a fourth power modulo $q)$;

(II) [10, Lemma 9.10.2]. $\left(\frac{b}{\pi}\right)_{4}=-1$ iff $b$ is a square but not a fourth power modulo $q$.

Lemma 2.2. Let $\pi=r+$ si be primary irreducible. Then we have

(I) $\left(\frac{-1}{\pi}\right)_{4}=(-1)^{\frac{r-1}{2}}[10$, Proposition 9.8.3(d)];

(II) $\left(\frac{2}{\pi}\right)_{4}=i^{\frac{r s}{2}}$ [9, Theorem 4.23]; [10, Exercise 5.27].

We also need the following three lemmas, the proofs of which are easy.

Lemma 2.3. Let $b$ be prime $\equiv 3 \bmod 4$ and $\pi=r+$ si be primary irreducible. Then we have

$$
\left(\frac{b}{\pi}\right)_{4}=(-1)^{\frac{r-1}{2}}\left(\frac{\pi}{b}\right)_{4} .
$$

Lemma 2.4. Let $\beta$ and $\pi$ be primary irreducible with different prime norms $\equiv 1$ $\bmod 4$. If $b=N(\beta)$, then we have

$$
\left(\frac{b}{\pi}\right)_{4}=\left(\frac{\pi \overline{\pi^{-1}}}{\beta}\right)_{4},
$$

where $\pi^{-1}$ denotes the inverse of $\pi$ modulo $b$.

Lemma 2.5. Let $\beta=u+v i$ be primary irreducible with prime $b=N(\beta) \equiv 1$ $\bmod 4$ and $\alpha=c+d i$. Then we have

$$
\left(\frac{\alpha}{\beta}\right)_{4} \equiv\left(c-d u v^{-1}\right)^{(b-1) / 4} \bmod b
$$

\section{K2-STRONG PSEUdopRIMES}

Throughout this section let $\pi$ be a primary irreducible of $D$ such that $q=$ $N(\pi) \equiv 1 \bmod 4$ and $p=(q+1) / 2$ are two primes determined by $\pi$. We are going to describe a method to compute all composite numbers $n=p q$, below a given limit (say $10^{24}$ ), which are strong pseudoprimes to the first several (say 10) prime bases. For this purpose we are looking for necessary conditions on $\pi$ for $n=p q$ to be a strong pseudoprime, first to a prime base $b$, then to several prime bases.

Proposition 3.1. If $n=p q$ is a strong pseudoprime to a (not necessarily prime) base $b$, then

$$
\left(\frac{b}{\pi}\right)_{4}=\left(\frac{b}{p}\right) .
$$

Proof. If $n=p q$ is an $\operatorname{spsp}(b)$ then, by Lemma 1.1,

$$
v_{2}\left(\operatorname{ord}_{p}(b)\right)=v_{2}\left(\operatorname{ord}_{q}(b)\right) .
$$


If $\left(\frac{b}{p}\right)=1$, then $v_{2}\left(\operatorname{ord}_{q}(b)\right)=v_{2}\left(\operatorname{ord}_{p}(b)\right) \leq v_{2}(p-1)-1=v_{2}(q-1)-2$; thus $b$ is a fourth power modulo $q$, and so, by Lemma $2.1(\mathrm{I})$,

$$
\left(\frac{b}{\pi}\right)_{4}=1=\left(\frac{b}{p}\right) \text {. }
$$

If $\left(\frac{b}{p}\right)=-1$, then $v_{2}\left(\operatorname{ord}_{q}(b)\right)=v_{2}\left(\operatorname{ord}_{p}(b)\right)=v_{2}(p-1)=v_{2}(q-1)-1$; thus $b$ is a square but not a fourth power modulo $q$, and so

$$
\left(\frac{b}{\pi}\right)_{4}=-1=\left(\frac{b}{p}\right)
$$

by Lemma 2.1(II).

For the rest of this section, let $p_{\alpha}=(N(\alpha)+1) / 2$ be a positive integer determined by a primary (but not necessarily irreducible) element $\alpha$ of $D$. If a prime $b \equiv 1$ $\bmod 4$, then $b=\beta \bar{\beta}$ for some primary irreducible $\beta$. If $\operatorname{gcd}(b, N(\alpha))=1$, then $\alpha^{-1}$ denotes the inverse of $\alpha$ modulo $b$.

Let

$$
R_{2}=\left\{\text { primary } \alpha=x+y i: 0 \leq x, y<8, i^{\frac{x y}{2}}=(-1)^{\frac{p_{\alpha}^{2}-1}{8}}\right\}=\{1,5+4 i\} .
$$

By Lemma 2.2(II) and Proposition 3.1 we have

Lemma 3.1. If $n=p q$ is an $\operatorname{spsp}(2)$, then there exists $\alpha \in R_{2}$ such that $\pi \equiv \alpha$ $\bmod 8$.

For a prime $b \equiv 3 \bmod 4$, let

$$
R_{b}=\left\{\alpha=x+y i: 0 \leq x, y<4 b, \alpha \equiv 1 \bmod 4,\left(\frac{\alpha}{b}\right)_{4}=\left(\frac{p_{\alpha}}{b}\right)\right\} ;
$$

and for a prime $b \equiv 1 \bmod 4$, let

$$
R_{b}=\left\{\alpha=x+y i: 0 \leq x, y<4 b, \alpha \equiv 1 \bmod 4,\left(\frac{\alpha \overline{\alpha^{-1}}}{\beta}\right)_{4}=\left(\frac{p_{\alpha}}{b}\right)\right\} .
$$

Using (2.3) for $b \equiv 3 \bmod 4$ and Lemma 2.5 for $b \equiv 1 \bmod 4$, it is easy to compute the sets:

$$
\begin{gathered}
R_{3}=\{1,5\} ; \quad R_{5}=\{1,9\} ; \\
R_{7}=\{1,13,21+8 i, 21+20 i, 1+8 i, 1+20 i, 13+8 i, 13+20 i, \\
17+4 i, 17+24 i, 25+4 i, 25+24 i\} ; \\
R_{11}=\{1,5+8 i, 5+36 i, 9+12 i, 9+32 i, 13+12 i, 13+32 i, 17+8 i, \\
17+36 i, 21,25,29,29+20 i, 29+24 i, 37,37+20 i, \\
37+24 i, 41,5+12 i, 5+32 i, 17+12 i, 17+32 i, 29+8 i, \\
29+36 i, 33+16 i, 33+20 i, 33+24 i, 33+28 i, 37+8 i, 37+36 i\} ;
\end{gathered}
$$

and

$$
\begin{aligned}
R_{13}=\{1,1+4 i, 1+48 i, 25,25+4 i, 25+48 i, 29+12 i, 29+40 i, \\
33,33+24 i, 33+28 i, 37,41,45,45+24 i, 45+28 i, \\
49+12 i, 49+40 i, 5+24 i, 5+28 i, 13+4 i, 13+16 i, 13+36 i, 13+48 i, \\
21+24 i, 21+28 i, 33+8 i, 33+44 i, 45+8 i, 45+44 i\} .
\end{aligned}
$$

By Lemmas 2.3 and 2.4 and Proposition 3.1 we have 
Lemma 3.2. Let $b$ be an odd prime. If $n=p q$ is an $\operatorname{spsp}(b)$ and $\pi \equiv 1 \bmod 4$, then there exists $\alpha \in R_{b}$ such that $\pi \equiv \alpha \bmod 4 b$.

Let $m=4 \cdot 2 \cdot 3 \cdot 5 \cdot 7 \cdot 11 \cdot 13=120120$. Applying the Chinese Remainder Theorem, it is easy to compute the set

$$
R=\left\{x+y i: 0 \leq x, y<m, x+y i(\bmod 4 b) \in R_{b} \text { for } b=2,3,5,7,11 \text { and } 13\right\},
$$

which has cardinality $\# R=2 \cdot 2 \cdot 2 \cdot 12 \cdot 30 \cdot 30=86400$. By Lemmas 3.1 and 3.2 and the Chinese Remainder Theorem we have

Proposition 3.2. If $n=p q$ is an spsp to the bases $2,3,5,7,11$ and 13 , then there exists $\alpha \in R$ such that $\pi \equiv \alpha \bmod m$.

Now we are ready to describe a procedure to compute all K2-spsp's $<L$, to the first $h(\geq 6)$ prime bases.

\section{PROCEDURE Finding-K2-spsp; \\ BEGIN}

For every $x+y i \in R, u \geq 0, v \geq 0, u+v \leq \frac{\sqrt[4]{8 L}}{m}+1$ Do

begin

$q \leftarrow(x+u m)^{2}+(y+v m)^{2} ; p \leftarrow(q+1) / 2 ; n \leftarrow p \cdot q ;$

If $n$ is an spsp to the first $h$ prime bases then output $n, p$ and $q$;

$q \leftarrow(x-u m)^{2}+(y+v m)^{2} ; p \leftarrow(q+1) / 2 ; n \leftarrow p \cdot q ;$

If $n$ is an spsp to the first $h$ prime bases then output $n, p$ and $q$; end

END.

The Pascal program (with multi-precision package partially written in Assembly language) ran about 33 hours on my PC486/66 to get all K2-spsp's $<10^{24}$ to the bases $2,3,5,7,11,13,17,19,23$, and 29 , listed in Table 1 . There are in total 41 numbers, among which six numbers are $\operatorname{spsp}(31)$, and three numbers are spsp's to both bases 31 and 37 .

\section{Example 3.1.}

$$
n=N_{10}=1955097530374556503981=31265776261 \cdot 62531552521
$$

is the smallest K2-spsp to the first 10 prime bases.

$$
\begin{gathered}
q=225739^{2}+107580^{2}, \quad \pi=-225739+107580 i \equiv 14501+107580 i \bmod m, \\
\left(\frac{b}{\pi}\right)_{4}=\left(\frac{b}{p}\right)= \begin{cases}-1, & \text { for } b=2,7,13,19 \text { and } 29 ; \\
1, & \text { for } b=3,5,11,17 \text { and } 23 .\end{cases}
\end{gathered}
$$

Remark 3.1. The 22-digit number $N_{10}$ in Example 3.1 yields the lowered upper bound for $\psi_{10}$. The old bound $M_{10}$ in $\S 1$ has 28 decimal digits, which is a K3-spsp found by Jaeschke [11], where about $\frac{22754930352733}{892371480} \cdot 400 \approx 1.02 \cdot 10^{7}$ cadidates were tested. If the method of Jaeschke [11] for finding large K2-spsp's had been used for finding the number $N_{10}$, about $\frac{31265776261}{4620} \cdot 15 \approx 1.015 \cdot 10^{8}$ candidates would have subjected to the Miller tests. With our method less than $86400 \cdot 6 \approx 5.184 \cdot 10^{5}$ candidates were tested, and the whole calculation took about 40 minutes on my $\mathrm{PC} 486 / 66$.

Remark 3.2. The only example spsp to the first 10 prime bases given in Arnault [4] has 46 decimal digits. 
TABLE 1. List of all K2-spsp's $<10^{24}$ to the first 10 prime bases

\begin{tabular}{|c|c|c|c|c|}
\hline \multirow[b]{2}{*}{ number } & \multirow[b]{2}{*}{ factorization } & \multicolumn{3}{|c|}{ spsp-base } \\
\hline & & 31 & 37 & 41 \\
\hline 1955097530374556503981 & 31265776261 & 0 & 0 & 0 \\
\hline 2690332968256326725221 & 36676511341 & 0 & 0 & 0 \\
\hline 3008407169465866853821 & 38784063541 & 0 & 0 & 1 \\
\hline 7395010240794120709381 & $60807114061 \cdot 121614228121$ & 1 & 0 & 0 \\
\hline 8322676924684188070621 & $64508437141 \cdot 129016874281$ & 0 & 0 & 0 \\
\hline 9363077444494472257261 & $68421770821 \cdot 136843541641$ & 0 & 0 & 0 \\
\hline 14677609160350387689301 & $85666823101 \cdot 171333646201$ & 0 & 0 & 0 \\
\hline 20403862733298647400661 & $101004610621 \cdot 202009221241$ & 0 & 0 & 1 \\
\hline 43832302471716234754141 & $148041045781 \cdot 296082091561$ & 0 & 0 & 0 \\
\hline 53174906711723993213881 & $163056595561 \cdot 326113191121$ & 0 & 1 & 0 \\
\hline 55571792871197983341781 & $166691020861 \cdot 333382041721$ & 0 & 0 & 0 \\
\hline 63251216294243798286901 & $177835902301 \cdot 355671804601$ & 0 & 0 & 1 \\
\hline 66990115045864781688781 & $183016549861 \cdot 366033099721$ & 0 & 0 & 1 \\
\hline 74900686097492114932621 & $193520911141 \cdot 387041822281$ & 0 & 0 & 0 \\
\hline 81867072311110130740741 & $202320379981 \cdot 404640759961$ & 0 & 1 & 0 \\
\hline 85985927737923413798461 & $207347447221 \cdot 414694894441$ & 0 & 1 & 1 \\
\hline 906137525501 & $217331008501 \cdot 434662017001$ & 0 & 0 & 0 \\
\hline 48927630751301 & $258993977101 \cdot 517987954201$ & 0 & 0 & 0 \\
\hline 3672633986221 & $286600958341 \cdot 573201916681$ & 1 & 0 & 0 \\
\hline 789138283962861 & $290027614021 \cdot 580055228041$ & 0 & 0 & 1 \\
\hline 964083744455945381 & $290685366061 \cdot 581370732121$ & 0 & 0 & 0 \\
\hline 247955734307378767707901 & $352104909301 \cdot 704209818601$ & 0 & 0 & 0 \\
\hline 115348395356661 & $395958142621 \cdot 791916285241$ & 0 & 0 & 0 \\
\hline 318665857834031151167461 & $399165290221 \cdot 798330580441$ & 1 & 1 & 0 \\
\hline 360681321802296925566181 & $424665351661 \cdot 849330703321$ & 1 & 0 & 0 \\
\hline 22748133501 & $431262544501 \cdot 862525089001$ & 0 & 0 & 0 \\
\hline 671685653341 & $435729756181 \cdot 871459512361$ & 0 & 0 & 0 \\
\hline 39820 & $446209834621 \cdot 8924$ & 0 & 1 & 0 \\
\hline 44959 & $474129500941 \cdot 94825900$ & 0 & 0 & 0 \\
\hline 500816 & $500408175181 \cdot 1000816$ & 0 & 1 & 0 \\
\hline 94556181 & $525703281661 \cdot 105140656$ & 1 & 1 & 0 \\
\hline 504737021 & $544171709941 \cdot 1088343$ & 0 & 0 & 1 \\
\hline 667636712015520329618581 & $577770158461 \cdot 1155540316921$ & 1 & 1 & 0 \\
\hline 767218076271537719164381 & $619361799061 \cdot 1238723598121$ & 0 & 1 & 0 \\
\hline 787275625773786753842101 & $627405620701 \cdot 1254811241401$ & 0 & 0 & 0 \\
\hline 804312708555704182158061 & $634157988421 \cdot 1268315976841$ & 0 & 0 & 0 \\
\hline 885194158945177223165341 & $665279700181 \cdot 1330559400361$ & 0 & 0 & 0 \\
\hline 919429103516313718344061 & $678022530421 \cdot 1356045060841$ & 0 & 0 & 1 \\
\hline 959800605007619741491261 & $692748368821 \cdot 1385496737641$ & 0 & 0 & 0 \\
\hline 959872525439982764342041 & $692774323081 \cdot 1385548646161$ & 0 & 0 & 0 \\
\hline 995879862373950674887861 & $705648589021 \cdot 1411297178041$ & 0 & 0 & 1 \\
\hline
\end{tabular}


TABLE 2. List of all K4-spsp's $<10^{24}$ to the first 9 prime bases

\begin{tabular}{|c|c|c|c|c|}
\hline & & \multicolumn{3}{|c|}{ spsp-base } \\
\cline { 3 - 5 } number & factorization & 29 & 31 & 37 \\
\hline 1683218926987824973501 & $20513525581 \cdot 82054102321$ & 0 & 0 & 0 \\
22816011932095901892127 & $75524850103 \cdot 302099400409$ & 0 & 0 & 0 \\
53050154675731441089751 & $115163095951 \cdot 460652383801$ & 0 & 0 & 0 \\
62226037292690827434451 & $124725736411 \cdot 498902945641$ & 0 & 0 & 1 \\
74615609992194444540751 & $136579290151 \cdot 546317160601$ & 0 & 0 & 0 \\
75627207586997811482107 & $137502006883 \cdot 550008027529$ & 0 & 0 & 0 \\
88390841560813963877707 & $148652986483 \cdot 594611945929$ & 0 & 0 & 0 \\
180639110735640469792951 & $212508300271 \cdot 850033201081$ & 0 & 1 & 0 \\
190724964865903509771451 & $218360347171 \cdot 873441388681$ & 0 & 1 & 0 \\
200726785895547132616207 & $224012714983 \cdot 896050859929$ & 0 & 0 & 0 \\
287772220090667093616451 & $268222025611 \cdot 1072888102441$ & 0 & 0 & 0 \\
372098606182110248554387 & $304999428763 \cdot 1219997715049$ & 0 & 0 & 0 \\
791375505322009804077727 & $444796443703 \cdot 1779185774809$ & 0 & 0 & 0 \\
939109651144284847866451 & $484538350171 \cdot 1938153400681$ & 1 & 0 & 1 \\
\hline
\end{tabular}

\section{K4-STRONG PSEUDOPRIMES}

To compute all composite numbers $n=p q$ below a given limit, of the form (1.3), with $k=4$ and $q=N(\pi)$ for some primary irreducible $\pi$ of $D$, which are strong pseudoprimes to the first several prime bases, the procedure is a little different from the case $k=2$. We give equivalent conditions on $\pi$ for $n$ to be a psp (instead of an spsp) to one or several prime bases. We subject those candidates $n$, with $\pi$ satifying the conditions, to Miller tests to decide whether they are spsp's or not.

Let $b$ be a positive integer (not necessarily prime). It is easy to prove that

$$
n=p q \text { is } \operatorname{asp}(b) \text { iff }\left(\frac{b}{\pi}\right)_{4}=1 .
$$

A procedure based on (4.1), lemmas in $\S 2$ and the Chinese Remainder Theorem ran about 61 hours on my PC486/66 to get all K4-spsp's $<10^{24}$ to the first 9 prime bases up to 23 , listed in Table 2 . There are in total 14 numbers, among which only one is $\operatorname{spsp}(29)$.

\section{Cubic Residue Characters And K3-Strong PSeudoprimes}

In this section $D$ denotes the ring

$$
\mathbb{Z}[\omega]=\{x+y \omega: x, y \in \mathbb{Z}\},
$$

where $\omega=\frac{-1+\sqrt{-3}}{2}$. It is well-known that $D$ is a Euclidean domain. Let $\alpha, \beta, \pi \in D$. The norm of $\alpha=x+y \omega$ is $N(\alpha)=\alpha \bar{\alpha}=x^{2}-x y+y^{2}$. The units in $D$ are the only six elements with norm $1: \pm 1, \pm \omega, \pm \omega^{2}$. The irreducibles of $D$ are $\pm(1-\omega), \pm(1+2 \omega), \pm(2+\omega)$ with norm 3 ; primes $\equiv 2(\bmod 3)$ and their associates; and non-real elements with prime norm $\equiv 1(\bmod 3)$. A prime $\equiv 1(\bmod 3)$ must be the norm of an irreducible of $D$; and the prime $3=-\omega^{2}(1-\omega)^{2}$. 
TABLE 3. List of all K3-spsp's $<10^{24}$ to the first 9 prime bases

\begin{tabular}{|c|c|c|c|c|}
\hline & & \multicolumn{3}{|c|}{ spsp-base } \\
\cline { 3 - 5 } number & factorization & 29 & 31 & 37 \\
\hline 1912984162541380673761 & $25251958093 \cdot 75755874277$ & 0 & 0 & 1 \\
34096886969005185504481 & $106609704013 \cdot 319829112037$ & 0 & 0 & 0 \\
55980401347534279735681 & $136602100213 \cdot 409806300637$ & 0 & 0 & 0 \\
197694930977378358089281 & $256706662021 \cdot 770119986061$ & 0 & 0 & 0 \\
236194333868452768765441 & $280591241173 \cdot 841773723517$ & 0 & 0 & 0 \\
359007177345502640908801 & $345932159701 \cdot 1037796479101$ & 0 & 0 & 1 \\
475946318312442156484321 & $398307384781 \cdot 1194922154341$ & 0 & 0 & 0 \\
584891146059350924824801 & $441546957133 \cdot 1324640871397$ & 0 & 0 & 0 \\
597958575967570275162721 & $446452153453 \cdot 1339356460357$ & 1 & 0 & 0 \\
609109964849973759917761 & $450595888741 \cdot 1351787666221$ & 1 & 0 & 1 \\
692926578711867648899521 & $480599132581 \cdot 1441797397741$ & 0 & 0 & 0 \\
\hline
\end{tabular}

A nonunit $\alpha$ is called primary if $\alpha \equiv 2(\bmod 3)$. Among six associates of a nonunit $\alpha$ satisfying $(1-\omega) \nmid \alpha$, there is (only) one which is primary. If $\pi$ is an irreducible with $N(\pi) \neq 3$ and $\pi \nmid \alpha$, there is a unique integer $m=0,1$, or 2 such that $\alpha^{(N(\pi)-1) / 3} \equiv \omega^{m} \bmod \pi$. The cubic residue character of $\alpha$ modulo $\pi$, with $N(\pi) \neq 3$ and $\pi \nmid \alpha$, is defined and denoted by $\left(\frac{\alpha}{\pi}\right)_{3}=\omega^{m}$, which is $1, \omega$ or $\omega^{2}=-1-\omega$. If $\pi \mid \alpha$, then $\left(\frac{\alpha}{\pi}\right)_{3}=0$. We have $\left(\frac{2}{\pi}\right)_{3}=1$ iff $\pi \equiv 1 \bmod 2[10$. Proposition 9.1].

Let $\pi$ be primary irreducible with prime $q=N(\pi) \equiv 1 \bmod 3$. Let $b \in \mathbb{Z}$ with $q \nmid b$. Then we have $\left(\frac{b}{\pi}\right)_{4}=1$ iff $x^{3} \equiv b \bmod q$ has a solution with $x \in \mathbb{Z}$, i.e., iff $b$ is a cubic residue modulo $q$ [10, Proposition 9.3.3(a)].

Let $\pi$ and $\beta$ be primary irreducibles with $N(\pi) \neq 3, N(\beta) \neq 3$, and $N(\pi) \neq N(\beta)$. Then $\left(\frac{\beta}{\pi}\right)_{3}=\left(\frac{\pi}{\beta}\right)_{3}$ (The law of cubic reciprocity [10, Theorem 9.1]).

Suppose that $N(\pi) \neq 3$. If $\pi=q$ is rational, write $q=3 m-1$; if $\pi=u+v \omega$ is a primary complex irreducible, write $u=3 m-1$. Then we have $\left(\frac{1-\omega}{\pi}\right)_{3}=\omega^{2 m}$ (Supplemment to the Cubic Reciprocity Law [10, Theorem 9.1']).

Let $\pi$ be a primary irreducible of $D$, and $q=N(\pi) \equiv 1 \bmod 3$ and $p=$ $(q+2) / 3$ two primes determined by $\pi$. It is easy to prove that if $n=p q$ is a strong pseudoprime to the (not necessarily prime) base $b$, then

$$
\left(\frac{b}{\pi}\right)_{3}=1 \quad \text { and } \quad\left(\frac{b}{p}\right)=\left(\frac{b}{q}\right) .
$$

A procedure based on (5.1), the Cubic Reciprocity Law and its Supplemment, and the Chinese Remainder Theorem ran about 8 hours on my PC486/66 to get all K3-spsp's $<10^{24}$ to the first 9 prime bases up to 23, listed in Table 3 . There are in total 11 numbers, among which only two are $\operatorname{spsp}(29)$ 's.

\section{ACKNOWLEDGMENTS}

I thank Professors F. Arnault, W. Bosma, A. K. Lenstra, H. W. Lenstra, Jr., C. Pomerance, G. Robin, and S. S. Wagstaff, Jr. for their sending me reprints or preprints concerning this subject or other topics in computational number theory. Special thanks go to the referee for helpful comments that improved the paper. 


\section{REFERENCES}

1. L.M.Adleman, C.Pomerance and R.S.Rumely, On distinguishing prime numbers from composite numbers., Annals of Math., 117 (1983), 173-206. MR 84e:10008

2. W.R.Alford, A.Granville and C.Pomerance, There are infinitely many Carmichael numbers, Annals of Math., 140 (1994), 703-722. MR 95k:11114

3. W.R.Alford, A.Granville and C.Pomerance, On the difficulty of finding reliable witnesses, Algorithmic Number Theory, pp.1-16, Lecture Notes in Computer Science, vol. 877, SpringerVerlag, Berlin, 1994. MR 96d:11136

4. F.Arnault, Rabin-Miller primality test: Composite numbers which pass it, Math. Comp., 64 (1995), 355-361. MR 95c:11152

5. A.O.L.Atkin and F.Morain, Elliptic curves and primality proving, Math. Comp., 61 (1993), 29-68. MR 93m:11136

6. W.Bosma and M.P. van der Hulst, Primality proving with cyclotomy, thesis, Univ. of Amsterdam, 1990.

7. H.Cohen and A.K.Lenstra, Implementation of a new primality test, Math. Comp., 48 (1987), 103-121. MR 88c:11080

8. H.Cohen and H.W.Lenstra,Jr., Primality testing and Jacobi sums, Math. Comp., 42 (1984), 297-330. MR 86g:11078

9. D.A.Cox, Primes of the form $x^{2}+n y^{2}$, Interscience, New York, 1989. MR 90m:11016

10. K.Ireland and M.Rosen, A classical introduction to modern number theory, Springer-Verlag, New York, 1982. MR 83g:12001

11. G.Jaeschke, On strong pseudoprimes to several bases, Math. Comp., 61 (1993),915-926. MR 94d:11004

12. G.Miller, Riemann's hypothesis and tests for primality, J.Comput. and System Sc., 13 (1976),300-317. MR 58:470a

13. Louis Monier, Evaluation and comparison of two efficient probabilistic primality testing algorithms, Theoretical Computer Science, 12 (1980), 97-108. MR 82a:68078

14. C.Pomerance, J.L.Selfridge and Samuel S.Wagstaff,Jr., The pseudoprimes to $25 \cdot 10^{9}$, Math. Comp., 35 (1980), 1003-1026. MR 82g:10030

15. M.O.Rabin, Probabilistic algorithms for testing primality, J.Number Theory, 12 (1980), 128138. MR 81f:10003

Department of Mathematics, Anhui Normal University, 241000 Wuhu, Anhui, P. R. CHINA

State Key Laboratory of Information Security, Graduate School USTC, 100039 BeiJING, P. R. ChINA

E-mail address: zhangzhx@mail.ahwhptt.net.cn 